\title{
High-Precision Control for Functional Electrical Stimulation Utilizing a High-Resolution Encoder
}

\author{
Sho Sakaino* \\ Naoto Mizukam \\ Tomoya Kitamura** \\ Student Member \\ Non-member, \\ Toshiaki Tsuji* \\ Senior Member
}

(Manuscript received April 27, 2020, revised Aug. 5, 2020)

J-STAGE Advance published date : Oct. 30, 2020

\begin{abstract}
In functional electrical stimulation, the time delay between an input voltage and the corresponding muscle force is a significant issue. This study revealed that the joint angle/voltage relationship can be modeled as a fourth-order system. This enables the inclusion of a time delay in the high-order phase delay of the force/voltage relationship, which may lead to delay recovery using a suitable controller. Accordingly, a full state feedback controller is proposed in this study, to recover the phase delays. Jerk measurement is mandatory for full state feedback controllers owing to the joint the fourth-order joint angle/voltage relationship. This issue can be solved by the recent development of high-resolution encoders. Finally, the validity of the proposed method was verified experimentally.
\end{abstract}

Keywords: functional electrical stimulation, high-resolution encoder, full state feedback control, position control

\section{Introduction}

Functional electrical stimulation (FES) is a technique that drives muscles utilizing outer current ${ }^{(1)(2)}$. Consequently, muscles can be driven regardless of subject consciousness. Over the past few decades, FES has been widely applied to facilitate various tasks in the field of medical engineering, such as object grasping ${ }^{(3)(4)}$, standing up ${ }^{(5)-(7)}$, cycling $^{(8)-(10)}$, and walking ${ }^{(1)(12)}$. Various methods to obtain command values for FES utilizing electroencephalogram signals have also been studied ${ }^{(13)-(15)}$. Notably, patient recovery has been the primary focus of medical applications, while control performance has been secondary. Therefore, most existing controllers are feedforward controllers that provide relatively poor control performance.

Recently, FES for healthy people has also received increased attention. Tamaki et al. demonstrated that FES can be utilized to teach skills for playing instruments ${ }^{(16)}$. Lopes et al. demonstrated that FES can be utilized to enhance virtual force ${ }^{(17)(18)}$. A haptic communication system utilizing FES was also proposed ${ }^{(19)-(21)}$. It should be noted that the goal of applying FES to healthy people is exclusively to control their bodies, not to achieve any medical effects. Therefore, the required specifications for control, such as robustness to modeling errors and control bandwidth, are much stricter for FES application to healthy people.

Two main problems of interest exist regarding FES control: time delay and nonlinearity. First, time delays are generated

\footnotetext{
${ }^{*}$ Faculty of Engineering, Information and Systems, Department of Intelligent Interaction Technologies, University of Tsukuba and JST, PRESTO

1-1-1, Tennodai, Tsukuba, Ibaraki, 305-8573, Japan

** Graduate School of Science and Engineering, Saitama University

255, Shimo-okubo, Sakura-ku, Saitama 338-8570, Japan
}

by the chemical reaction process that occurs during muscle contraction ${ }^{(22)}$. Because phase delays caused by time delays cannot be recovered by a controller, designers have primarily focused on stability enhancement ${ }^{(23)(24)}$. Second, the suppression of nonlinearity has been studied extensively. Controllers based on neural networks (NNs) have been developed to treat nonlinearities of muscle force ${ }^{(25)(26)}$. Sampson et al. improved position tracking accuracy utilizing iterative learning control ${ }^{(27)}$. However, this solution is not practical for use with healthy people owing to low robustness against disturbances. Recently, many researchers have improved FES robustness utilizing sliding mode control (SMC). The chattering phenomenon is problematic in SMC. Ajoudani et al. utilizing an NN to implement SMC while suppressing chattering ${ }^{(28)}$. Farhoud et al. implemented high-order SMC in which chattering was drastically reduced to achieve precise cycling motion ${ }^{(29)}$. Nekoukar et al. integrated SMC with fuzzy control ${ }^{(30)}$. Zhang et al. modeled and estimated muscle fatigue utilizing an evoked electromyograpy signal ${ }^{(31)}$. Control precision has been improved based on the implementation of these controllers. Yet, control bandwidth is still limited and joint movements in FES are slow. This is a result of the low feedback gains associated with phase delays caused by time delays. Without resolving the issue of phase delay, a wide control bandwidth for FES cannot be obtained.

When nonlinear terms are ignored, the force/voltage relationship in FES is modeled by a second-order system with time delay ${ }^{(32)(33)}$. Because the force/joint angle relationship is a single-inertia system, it is also a second-order system. Therefore, the joint angle/voltage relationship is a fourthorder system with time delay. To resolve the issue of phase delay, the contribution of time delays to phase delays must be determined. We must also determine if the fourth-order system or time delay are dominant.

In this study, we determined that the dominant phase delay 
for control is caused by the fourth-order system and that the phase delay of the time delay is negligible. Therefore, phase delay can be recovered utilizing a full state feedback controller. Alibeji et al. proposed a nonlinear controller to compensate for phase delays ${ }^{(34)}$. They modeled the force/voltage relationship as a first-order system. However it is actually a second-order system, as discussed in Section 3, and fast motion could not be obtained. A full state feedback controller designed based on a second-order force/voltage model and fourth-order joint angle/voltage model requires jerk responses. Consequently, no full state feedback controller has been utilized in FES to date.

In the field of mechatronics, fourth-order systems have been widely studied, particularly in two-mass resonant systems. Some typical examples are resonant ratio control ${ }^{(35)-(38)}$, full state feedback control ${ }^{(39)-(41)}$, and self-resonance cancellation techniques ${ }^{(42)-(44)}$. These controllers require only position, velocity, and acceleration responses. The measurement of jerk responses has traditionally been impractical and this problem has been largely avoided by utilizing multiple sensors. However, recent advancements in optical encoders have enabled jerk measurement. Yamada et al. demonstrated that full state feedback control can be achieved utilizing a highresolution encoder that measures jerk responses ${ }^{(45)}$. These results have significantly improved the practicality of full state feedback control.

In this study, advancements in mechatronics were integrated with FES. This paper proposes a full state feedback controller for FES utilizing a high-resolution encoder for measurements, including jerk responses. Although the proposed method does not include a nonlinear compensator, such as SMC, fast and accurate control is obtained by recovering phase delay. In summary, the following two conclusions can be drawn regarding FES based on this study:

(1) Time delay is not a dominant cause of phase delay.

(2) Full state feedback control is applicable when utilizing high-resolution encoders.

The validity of these conclusions was verified experimentally.

The remainder of this paper is organized as follows. Section 2 describes FES. In Section 3, an FES model is presented and its parameters are identified. Section 4 proposes a full state feedback controller. Its validity is established via simulations and experiments in Sections 5 and 6. Finally, our conclusions are presented in Section 7.

\section{Functional Electrical Stimulation}

In this study, we focused on controlling elbow joints. Therefore, biceps and triceps brachii muscles were stimulated by outer currents. The stimulation locations (also called motor points) on the biceps and triceps brachii muscles are presented in Fig. 1. The motor points were identified utilizing the Compex Performance (Compex) and adhesive pads (dimensions: $50 \times 50 \mathrm{~mm}$, AXEL) were attached to the motor points.

Figure 2 presents an example stimulation waveform. A $50 \mathrm{~Hz}$ pulse wave with a $1 \mathrm{~ms}$ pulse width was applied to stimulate the muscles. Because both positive and negative voltages were applied alternatingly, DC current was suppressed to prevent burn injuries. In this research, we controlled the amplitude of voltage according to the reference ${ }^{(16)}$.

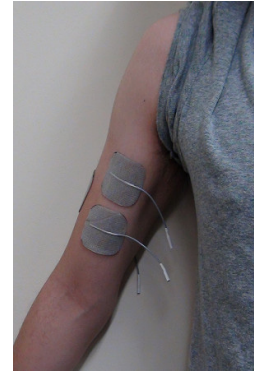

(a)

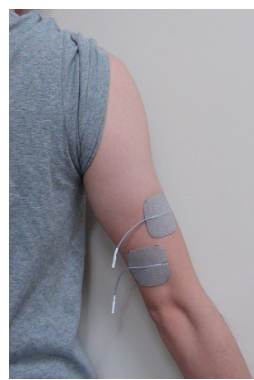

(b)
Fig. 1. Stimulation locations (motor points): (a) biceps brachii muscle (flexor muscle) and (b) triceps brachii muscle (extensor muscle)

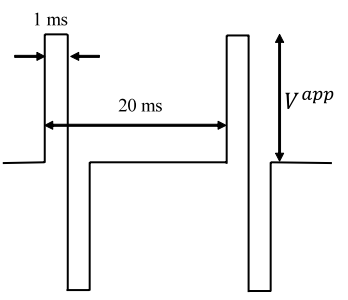

Fig. 2. Stimulation voltage

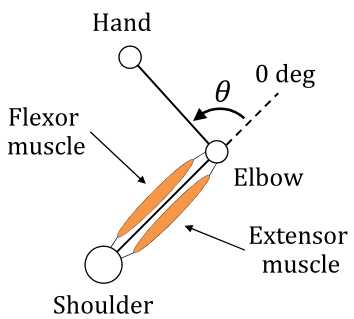

Fig. 3. Angle definition
For applications where fatigue is a particular problem, such as medical applications, stimulation at $20 \mathrm{~Hz}$ is often used. However, because this paper focuses most on control performance, $50 \mathrm{~Hz}$ was chosen because it had the best response performance in preliminary experiments.

In this study, the movements of muscles were controlled by adjusting the amplitude of the pulse wave, which is defined as $V^{a p p}$, and a voltage feedback controller was implemented to reject the effects of perturbation of electrical characteristics. A safety circuit was designed to limit the current within $20 \mathrm{~mA}$ to conform to the Japanese Industrial Standard. The measured effective value was less than $2 \mathrm{~mA}$, which was a very safe experiment. Additionally, the angle of the elbow joint, denoted $\theta$, was defined as shown in Fig. 3.

\section{FES Model}

First, the relationship between the input voltage and muscle torque was modeled. Figure 4 presents the experimental setup. In this experiment, the subjects placed their arms on a desk and effects of gravity were ignored. Next, their elbow and wrist joints were fixed while exerted tip force was measured utilizing a force sensor (PFS055YA251U6, Leptrino).

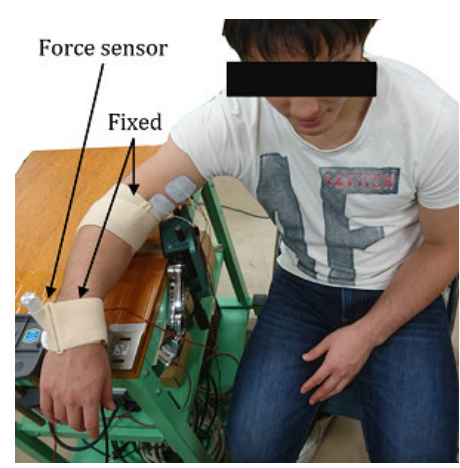

Fig. 4. Experiment for the force/voltage relationship 


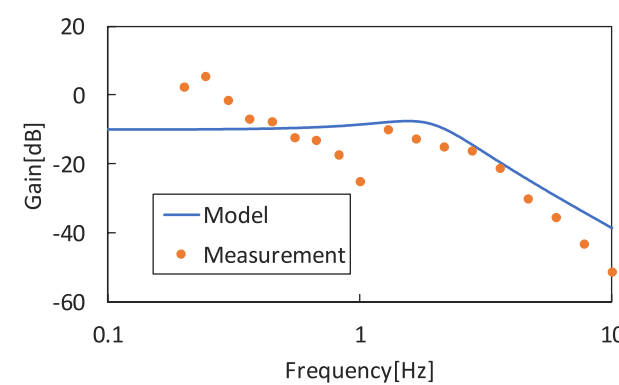

(a)

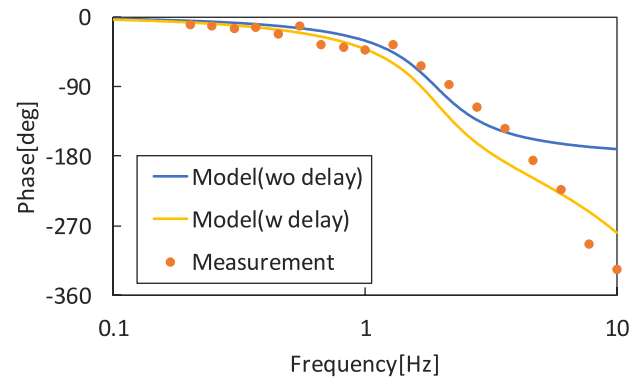

(b)

Fig. 5. Frequency characteristics (subject A, flexor) of (a) gain and (b) phase

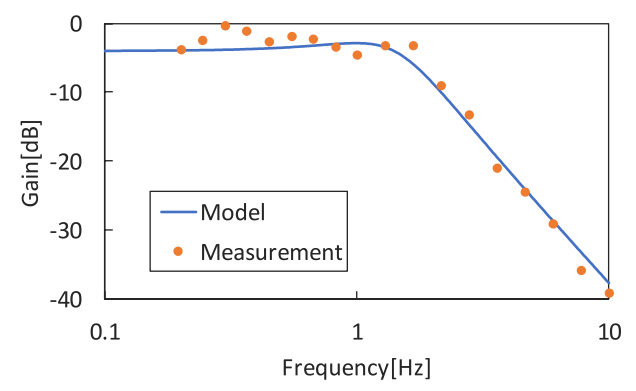

(a)

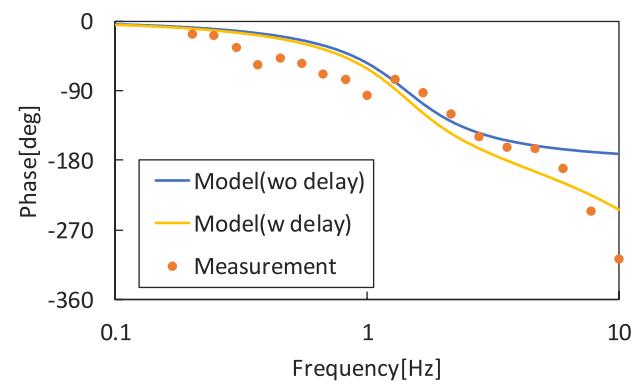

(b)

Fig. 6. Frequency characteristics (subject A, extensor) of (a) gain and (b) phase

Specifically, the elbow joint was fixed at $\theta=\frac{\pi}{4}$. Additionally, the subjects maintained contact with the force sensor at all times. It should be noted that the tip force $f$ was measured instead of the muscle torque $\tau$. These values are related as $\tau=f l$ with a moment arm of $l$.

The frequency responses of two healthy male subjects in their twenties, referred to as A and B, were obtained to model the force/voltage relationship. A minimum voltage $V^{\text {th }}$ was required to generate muscle torque during FES. These values were preliminarily identified for each subject's muscle such that $\hat{V}^{\text {th }}$ was the voltage with a force response of $1 \mathrm{~N}$. An input voltage without the bias voltage $V^{t h}, u$, was applied to obtain the frequency characteristics produced by a sine wave

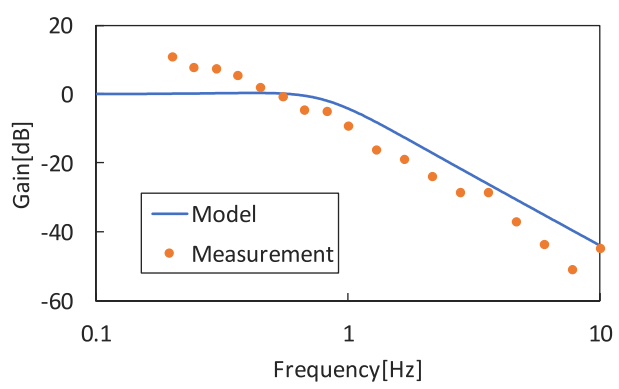

(a)

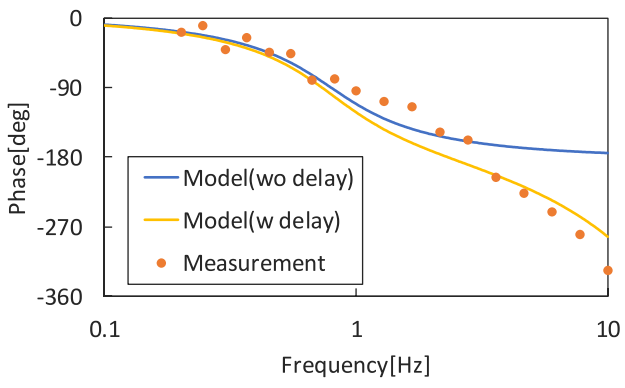

(b)

Fig. 7. Frequency characteristics (subject B, flexor) of (a) gain and (b) phase

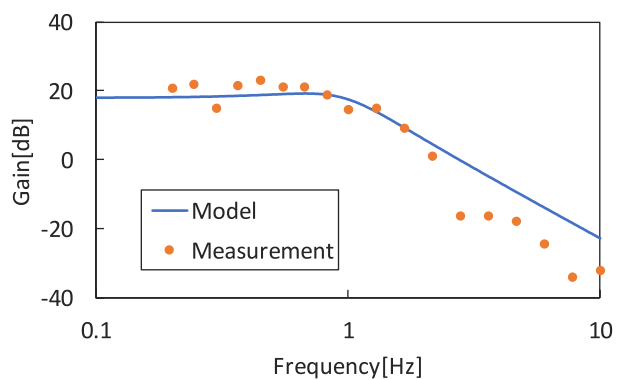

(a)

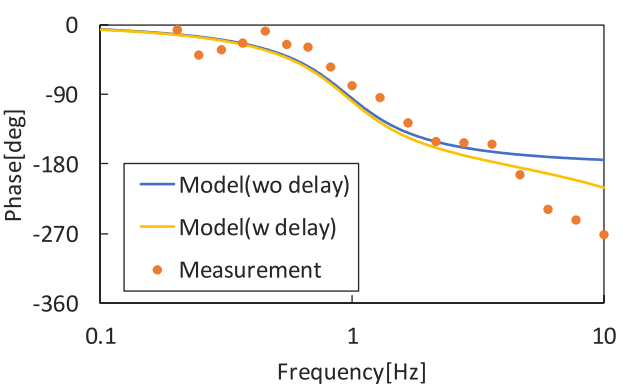

(b)

Fig. 8. Frequency characteristics (subject B, extensor) of (a) gain and (b) phase

sweep. Next, $V^{a p p}=u+\hat{V}^{t h}$ was applied to the muscles. Prior to our experiments, informed consent was obtained from all participants. Additionally, the Ethics Committees of Saitama University and University of Tsukuba approved all experiments conducted in this study.

The frequency characteristics of two muscles (a flexor and extensor of subject A and B) are presented in Figs. 5 to 8 . Orange dots represent responses and solid lines represent estimated models of the force/voltage relationship in the form of a second-order system based on the model discussed in ${ }^{(32)(33)}$. In the phase characteristics, models with and without time delay are expressed as yellow and blue solid lines, respectively. 
Table 1. Parameters of the force/torque relationship

\begin{tabular}{|lcccc|}
\hline Subject & $K$ & $\zeta$ & $\omega_{n}$ & $\mathrm{~T}$ \\
\hline A (flexor) & 0.31 & 0.41 & 12 & $0.03 \mathrm{~s}$ \\
A (extensor) & 0.63 & 0.51 & 9 & $0.02 \mathrm{~s}$ \\
B (flexor) & 1 & 0.6 & 5 & $0.03 \mathrm{~s}$ \\
B (extensor) & 7.94 & 0.5 & 18 & $0.01 \mathrm{~s}$ \\
\hline
\end{tabular}

Table 2. RMSE [deg] values for phase characteristics

\begin{tabular}{|lcc|}
\hline Subject & without delay & with delay \\
\hline A (flexor) & 10.9 & 17.4 \\
A (extensor) & 26.5 & 24.4 \\
B (flexor) & 10.4 & 13.9 \\
B (extensor) & 17.3 & 20.8 \\
\hline
\end{tabular}

The models can be formalized in (1),

$$
\frac{f}{u}=K \frac{\omega_{n}^{2}}{s^{2}+2 \zeta \omega_{n} s+\omega_{n}^{2}} e^{-s T} .
$$

where $K, \zeta, \omega_{n}$, and $T$ are the DC gain, damping coefficient, natural angular frequency, and time delay, respectively. In the case with no delay, $T=0$ is assumed while the other parameters remain the same. The identified parameters for each muscle are listed in Table 1. As shown in Figs. 5 to 8, the models agree well with the measured frequency responses in most frequency domains. However, differences between the models can be observed in the gain characteristics in the lowfrequency domain in Fig. 5(a) and Fig. 7(a) and in the phase characteristics in frequency domains greater than the natural frequencies. Because the identification of $V^{\text {th }}$ was difficult, errors in the gain responses were generated. Therefore, models should be identified based on phase responses. Table 2 presents root mean squared error (RMSE) lower than the natural frequencies showing that there was no significant difference. Notably, any motion faster than that of the natural frequencies is difficult to control, so most controllers output slower command values than natural frequencies. Modeling errors in such fast domains have few effects on overall control performance. Therefore, time delay does not play a dominant role in phase delay from the perspective of controller design.

\section{Full State Feedback Controller}

Figure 9 presents a block diagram of a musculoskeletal system. The dashed box represents the transfer function presented in the previous section. The parameters $m$ and $d$ represent the mass and damper of an arm, respectively. Here, the stiffness of muscles was neglected because reference ${ }^{(46)}$ demonstrated that precise dynamics models can be obtained without stiffness. If antagonistic muscles are activated, the stiffness of muscles cannot be neglected, but such concerns are beyond the scope of this study. Accordingly, the transfer function can be simplified in (2).

$$
\theta=\frac{b_{0}}{s^{4}+a_{3} s^{3}+a_{2} s^{2}+a_{1} s} u \cdots \cdots \cdots \cdots \cdots \cdots \cdots
$$

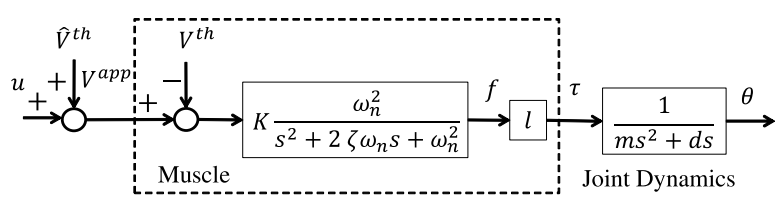

Fig. 9. Block diagram of a musculoskeletal system
This relationship can be expressed in a state space in (3) and (4),

$$
\begin{aligned}
& \frac{d}{d t} \boldsymbol{x}=\boldsymbol{A} \boldsymbol{x}+\boldsymbol{b} u, \\
& y=\theta=\boldsymbol{c x}, \cdots
\end{aligned}
$$

where the variables are defined as follows:

$$
\begin{aligned}
\boldsymbol{x} & =\left[\begin{array}{c}
\theta \\
\dot{\theta} \\
\ddot{\theta} \\
\dddot{\theta}
\end{array}\right], \quad \boldsymbol{A}=\left[\begin{array}{cccc}
0 & 1 & 0 & 0 \\
0 & 0 & 1 & 0 \\
0 & 0 & 0 & 1 \\
0 & -a_{1} & -a_{2} & -a_{3}
\end{array}\right], \\
\boldsymbol{b} & =\left[\begin{array}{c}
0 \\
0 \\
0 \\
b_{0}
\end{array}\right], \quad \boldsymbol{c}=\left[\begin{array}{llll}
1 & 0 & 0 & 0
\end{array}\right] .
\end{aligned}
$$

Based on the identified model, a full state feedback controller for FES systems is proposed. The proposed controller consists of a feedback controller $-\boldsymbol{f} \boldsymbol{x}$ to stabilize the system and a feedforward controller $C_{f f}$ to improve tracking performance. Here, $\boldsymbol{f}$ represents feedback gain, which is defined as $\boldsymbol{f}=\left[K_{p}, K_{v}, K_{a}, K_{j}\right]$. Equations (5) and (6) re obtained by substituting $u=-\boldsymbol{f} \boldsymbol{x}+C_{f f} \theta^{\text {cmd }}$ into (3):

$$
\begin{aligned}
& \frac{d}{d t} \boldsymbol{x}=(\boldsymbol{A}-\boldsymbol{b} \boldsymbol{f}) \boldsymbol{x}+\boldsymbol{b} C_{f f} \theta^{c m d} \ldots \\
& \theta=\boldsymbol{c}(s \boldsymbol{I}-\boldsymbol{A}+\boldsymbol{b} \boldsymbol{f})^{-1} \boldsymbol{b} C_{f f} \theta^{c m d} .
\end{aligned}
$$

Here, variables with the superscript "cmd" denotes command value. Because the feedback controller utilizes a feedback loop containing jerk responses, its implementation would not have been possible in the past. However, recent advancement in optical encoders have enabled the measurement of jerk responses. The proposed method exploits this ability of optical encoders. The feedforward controller was designed in (7) to obtain the transfer function $\frac{\theta}{\theta^{\text {cmd }}}=1$.

$$
C_{f f}=\left(\boldsymbol{c}(s \boldsymbol{I}-\boldsymbol{A}+\boldsymbol{b} \boldsymbol{f})^{-1} \boldsymbol{b}\right)^{-1} .
$$

Figure 10 presents a block diagram of the proposed method. Here, variables with the subscripts " $f$ " and " $e$ " represent variables for the flexor and extensor, respectively. The inputs for flexor and extensor muscles, namely $u_{f}$ and $u_{e}$, are determined in (8).

$$
\begin{aligned}
& u_{f}= \begin{cases}u & (u \geq 0) \\
0 & (u<0)\end{cases} \\
& u_{e}= \begin{cases}0 & (u \geq 0) \\
-u & (u<0)\end{cases}
\end{aligned}
$$

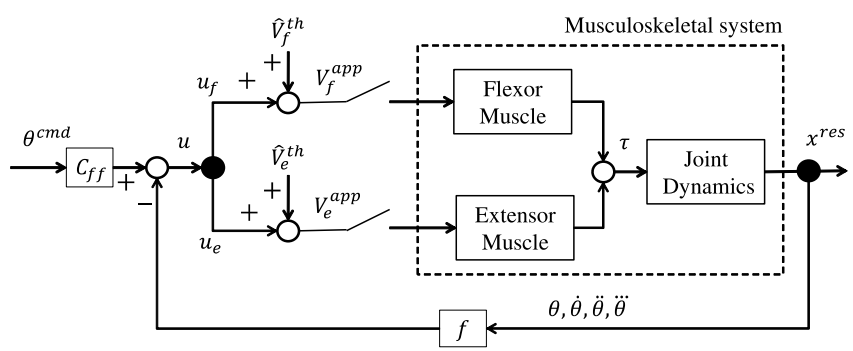

Fig. 10. Full state feedback controller 
Table 3. Simulation parameters

\begin{tabular}{|c|ccccc|}
\hline model & $V_{t h}[\mathrm{~V}]$ & $a_{1}$ & $a_{2}$ & $a_{3}$ & $b_{0}$ \\
\hline 4th order & 10 & 2000 & 400 & 30 & 15000 \\
2nd order & 10 & 5 & & & 37.5 \\
\hline
\end{tabular}

Table 4. Feedback gains in simulations

\begin{tabular}{|c|cccc|}
\hline model & $K_{p}$ & $K_{v}$ & $K_{a}$ & $K_{j}$ \\
\hline fourth-order & 26.0 & 4.03 & 0.223 & $4.67 \times 10^{-3}$ \\
second-order & 1.71 & 0.293 & & \\
\hline
\end{tabular}

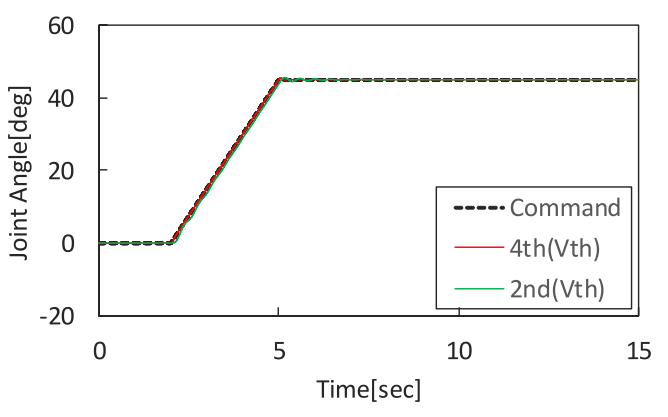

(a)

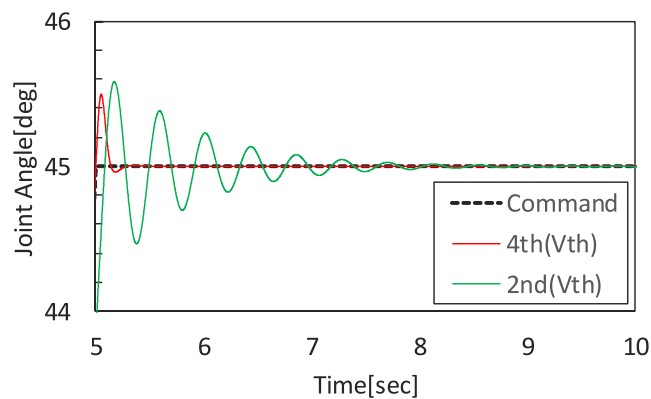

(b)

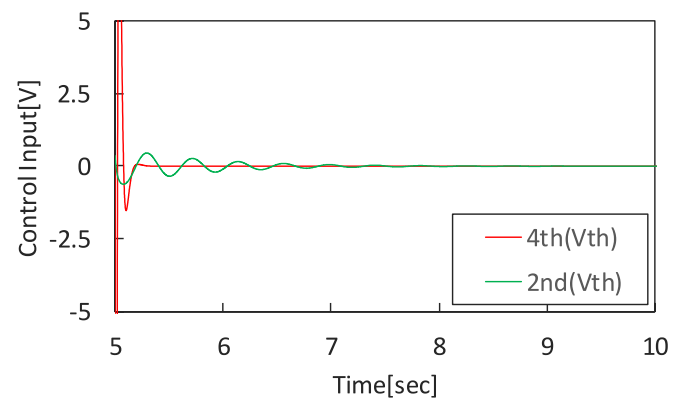

(c)

Fig. 11. Simulation results (without modeling error, $\hat{V}^{t h}=V^{t h}$ ): (a) position response, (b) magnified view of (a), and (c) control input

\section{Simulation}

First, the validity of the proposed method was verified via simulations. In our simulations, both the flexor and extensor muscles were designed to have the same parameters as those listed in Table 3. The conventional model expressed in (9) was also included for the sake of comparison.

$$
\theta=\frac{b_{0}}{s^{2}+a_{1} s} u
$$

For the conventional method, the proportional-derivative (PD) controller presented in (10) was adopted.

$$
u=K_{p}\left(\theta^{c m d}-\theta\right)+K_{v} s\left(\theta^{c m d}-\theta\right)+s^{2} \theta^{c m d}
$$

An acceleration feedforward term was implemented such that

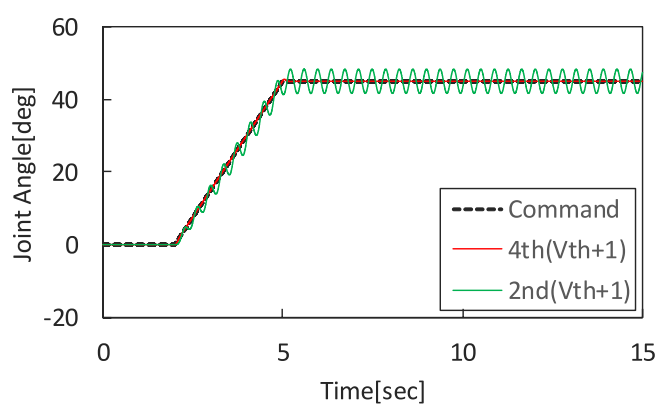

(a)

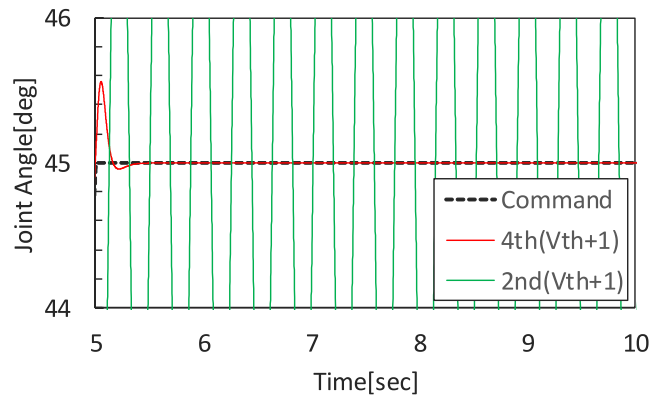

(b)

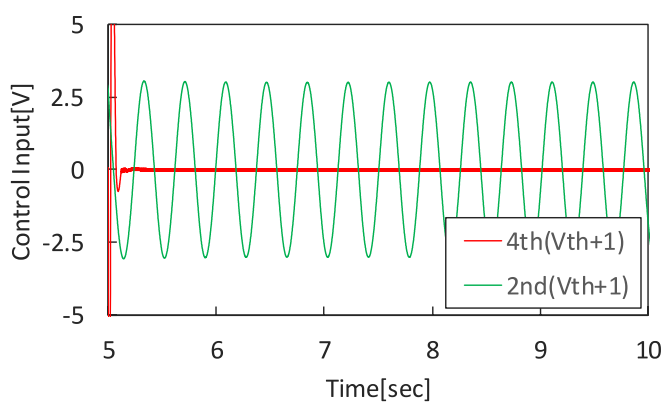

(c)

Fig. 12. Simulation results (with modeling error, $\hat{V}^{\text {th }}=$ $V^{\text {th }}+1$ ): (a) position response, (b) magnified view of (a), and (c) control input

the transfer function would have a value of one. Additionally, both the proposed and conventional methods were considered without integrators to simplify discussion. The feedback gains of the proposed method were designed to have a quadruple pole at $25 \mathrm{rad} / \mathrm{s}$, whereas those of the conventional method were designed to have a double pole at $8 \mathrm{rad} / \mathrm{s}$ to match the phase margin of the proposed method. Therefore, the proposed method can achieve a higher feedback gain with the same phase margin. Table 4 lists detailed feedback gains.

Figures 11 and 12 present the simulation results. Figure 11 presents a simulation result without any modeling error for the threshold value $\left(\hat{V}^{t h}=V^{t h}\right)$. As shown in Fig. 11, the responses of the proposed method (red line) and those of the conventional method (green line) both track the command values (black dotted line) accurately. However, Fig. 11(b) reveals that the responses of the proposed method converged faster. Because the poles of the proposed method can be greater than those of the conventional method, even with the same phase margin, the control performance of the proposed method is superior. As shown in Fig. 12, the difference in control performance was more evident in the case where we considered the modeling error of the threshold 
value $\left(\hat{V}^{t h}=V^{t h}+1\right)$. This is a reproduction of the fact that accurate estimation of $\mathrm{Vth}$ is difficult with an error of about $1 \mathrm{~V}$. This modeling error is equivalent to a disturbance voltage that equal to $1 \mathrm{~V}$ with $u \geq 0$ and $-1 \mathrm{~V}$ with $u<0$. As shown in Fig. 12(a), a chattering-like phenomenon was observed. However, even in this case, the proposed method only produced small errors, as shown in Fig. 12(b). Figure 12(c) presents the control inputs. Figures 12(b) and 12(c) clearly demonstrate that the proposed method was much faster than the conventional method, resulting in smaller amplitudes of cyclic waves.

\section{Experiments}

In this section, experimental verification of the proposed method was discussed. It should be noted that performing a fair comparison between nonlinear controllers is relatively difficult, whereas linear controllers can be compared easily by considering stability margins (gain margins and phase margins). Therefore, the proposed method was only compared to the PD controller as a representative linear controller.

Figure 13 presents a high-resolution encoder with 18-bit resolution (MAS-18, Microtech Laboratory Inc.). This encoder was attached to each subject's elbows utilizing two belts. The angular velocity, acceleration, jerk responses were obtained by using pseudo derivative with cut-off frequency of $20 \mathrm{rad} / \mathrm{s}$. The tracking performances of the proposed method and conventional PD controller were compared for four healthy male subjects in their twenties, who are referred to as C, D, E, and F. Similar to Section 3, informed consent was obtained from all participants. Because $V^{\text {th }}$ is actually a function of $\theta$, it is not a constant value, as assumed in Sections 3 and 5. Therefore, a more detailed model in the form of a quadratic function of $\theta$ was introduced in our experiments by referring to ${ }^{(47)}$.

$$
V^{\text {th }}=A_{2} \theta^{2}+A_{1} \theta+A_{0} \ldots \ldots \ldots \ldots \ldots \ldots \ldots
$$

To identify the parameters of $V^{\text {th }}, \theta$ was varied from $15^{\circ}$ to $75^{\circ}$ in $15^{\circ}$ increments and the applied voltage was increased in $0.5 \mathrm{~V}$ increments. The voltage was recorded just prior to the voltage at which the joint moved. Next, utilizing the least square method, the parameters were identified, as listed in Table 5.

Next, the parameters of the transfer function $\left(a_{1}, a_{2}, a_{3}, b_{0}\right)$ were then identified. However, this was difficult because when the direction of motion reversed, the contact conditions between the belts and arm changed. To eliminate this effect, identification was conducted by expanding the method proposed by Yamazaki et al. ${ }^{(46)}$. To identify the dynamics of flexor muscles, M-sequence signals given by $\hat{V}_{f}^{t h}$ and $\hat{V}_{f}^{t h}+5 \mathrm{~V}$ were applied to each subject's flexor muscles. Here, $V_{f}^{\text {th }}$ is a voltage threshold of flexor muscles and $\hat{V}_{f}^{\text {th }}$ is its estimate. Once each subject's arm reached the limit of the movable range, extensor muscles were identified in the same manner. Because motion data near the limit of the movable range are contaminated by changes in the contact conditions of the belts, such data were discarded. Additionally, the motion data were decimated and time windows of $50 \mathrm{~ms}$ were selected and filtered by a low-pass filter with a cutoff frequency of

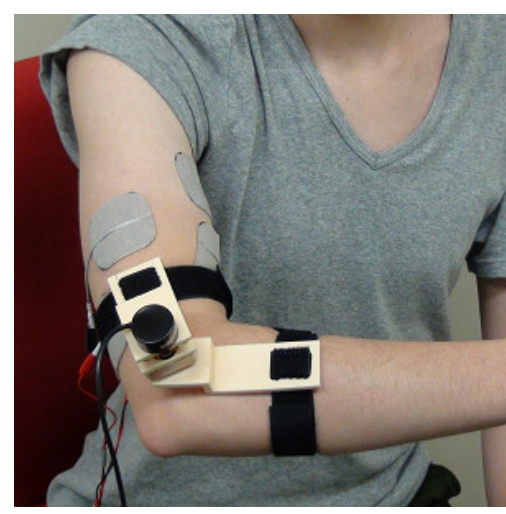

Fig. 13. High-resolution encoder

Table 5. Paramters of $V^{\text {th }}$

\begin{tabular}{|lccc|}
\hline Subject & $A_{0}$ & $A_{1}$ & $A_{2}$ \\
\hline C (flexor) & 7.10 & -5.54 & 3.65 \\
C (extensor) & 4.60 & -4.91 & 3.13 \\
D (flexor) & 9.00 & -4.53 & 3.13 \\
D (extensor) & 8.70 & -3.77 & 3.13 \\
E (flexor) & 6.70 & -8.29 & 6.25 \\
E (extensor) & 9.80 & -7.94 & 4.69 \\
F (flexor) & 6.60 & -8.02 & 7.29 \\
F (extensor) & 3.00 & 1.91 & 0.00 \\
\hline
\end{tabular}

Table 6. Parameters of musculoskeletal system (fourth order)

\begin{tabular}{|lcccc|}
\hline Subject & $a_{1}$ & $a_{2}$ & $a_{3}$ & $b_{0}$ \\
\hline C (flexor) & $1.79 \times 10^{5}$ & $1.28 \times 10^{4}$ & 15.1 & $1.50 \times 10^{5}$ \\
C (extensor) & $1.53 \times 10^{5}$ & $6.50 \times 10^{3}$ & 67.5 & $9.04 \times 10^{4}$ \\
D (flexor) & $7.30 \times 10^{4}$ & $3.39 \times 10^{3}$ & $1.64 \times 10^{2}$ & $3.02 \times 10^{4}$ \\
D (extensor) & $5.93 \times 10^{4}$ & $4.36 \times 10^{3}$ & 47.5 & $7.15 \times 10^{4}$ \\
E (flexor) & $6.35 \times 10^{4}$ & $6.07 \times 10^{3}$ & 11.2 & $7.11 \times 10^{4}$ \\
E (extensor) & $2.73 \times 10^{5}$ & $2.44 \times 10^{4}$ & $1.41 \times 10^{2}$ & $2.60 \times 10^{5}$ \\
F (flexor) & $1.92 \times 10^{5}$ & $2.60 \times 10^{4}$ & 48.1 & $1.42 \times 10^{5}$ \\
F (extensor) & $1.88 \times 10^{5}$ & $2.11 \times 10^{4}$ & 35.8 & $1.56 \times 10^{5}$ \\
\hline
\end{tabular}

Table 7. Parameters of musculoskeletal system (second order)

\begin{tabular}{|lcc|}
\hline Subject & $a_{1}$ & $b_{0}$ \\
\hline C (flexor) & 15.4 & 12.8 \\
C (extensor) & 32.3 & 19.9 \\
D (flexor) & 24.3 & 14.0 \\
D (extensor) & 15.1 & 20.8 \\
E (flexor) & 13.3 & 14.1 \\
E (extensor) & 11.2 & 11.1 \\
F (flexor) & 7.60 & 5.70 \\
F (extensor) & 9.80 & 7.90 \\
\hline
\end{tabular}

$10 \mathrm{~Hz}$. In the case of the second-order system, the parameters were identified in the same manner. Tables 6 and 7 list the parameters for each subject's flexor and extensor muscles.

In this study, the feedback gains of the proposed method (fourth-order model) were designed such that the transfer function for a flexor muscle had a quadruple pole at $25 \mathrm{rad} / \mathrm{s}$. Next, a transfer function for an extensor muscle was designed to have a quadruple pole such that the phase margin was the same as that for the flexor muscle. In the conventional method (second-order model), both flexor and extensor muscles are designed such that the phase margins are the same as that for the flexor muscle in the proposed method.

To avoid the effects of fatigue, all subjects took $5 \mathrm{~min}$ breaks between experiments. Three types of commands were given to the controller: a step command, trapezoidal 


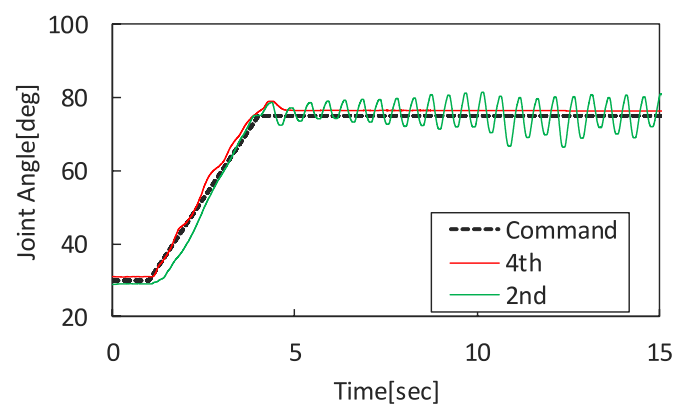

(a)

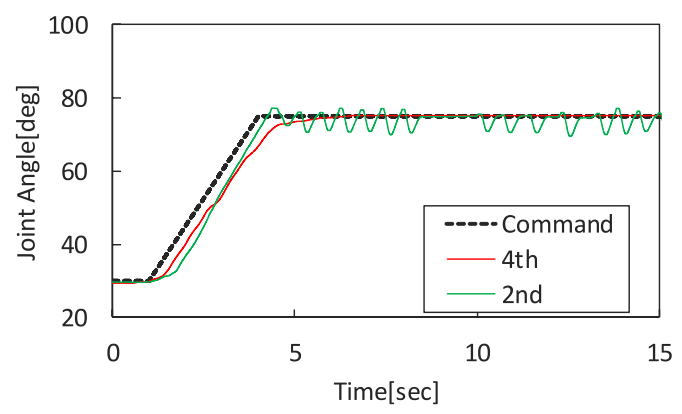

(b)

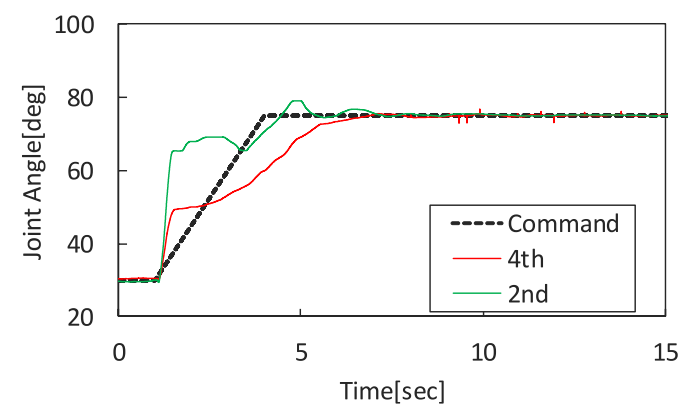

(c)

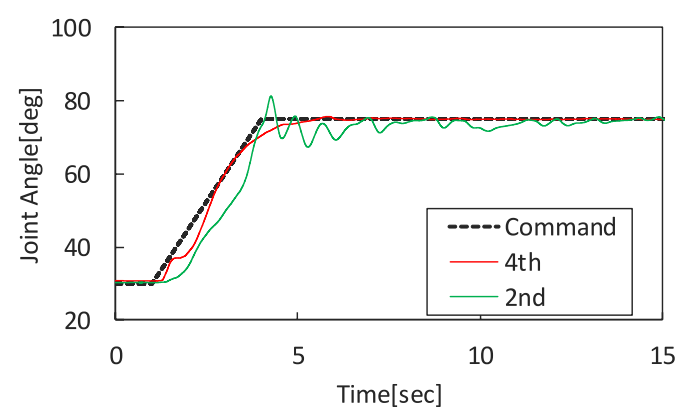

(d)

Fig. 14. Experimental results for the step command for (a) Subject C, (b) Subject D, (c) Subject E, and (d) Subject $F$

command, and synthetic sine waves with frequencies of 0.05 , $0.1,0.2$, and $0.7 \mathrm{~Hz}$. The experiment was conducted once for each command value. In other words, four subjects performed a total of 12 experiments. Figures 14, 15, and 16 present the results of the step command, trapezoidal command, and sine waves, respectively. Black dotted lines, red lines, and green lines represent commands, responses from the proposed method, and responses from the conventional method, respectively.

Figure 14 reveals good control performance for the proposed method, but an oscillatory steady state can be observed in the responses of the conventional method. These

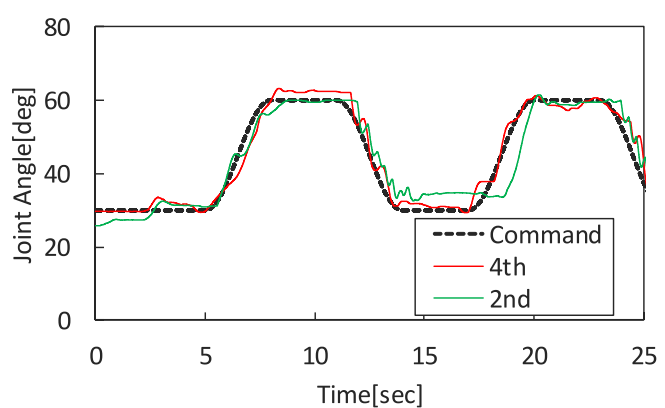

(a)

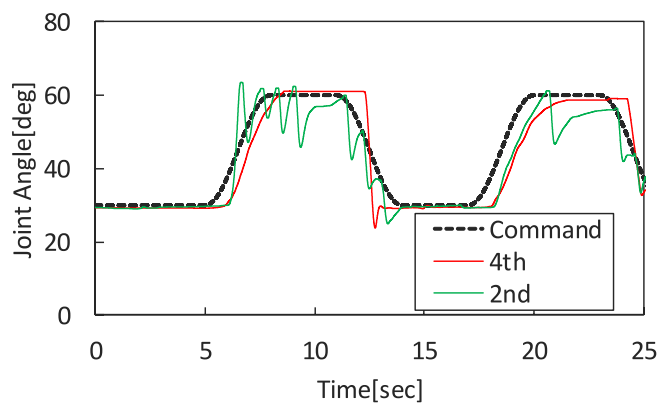

(b)

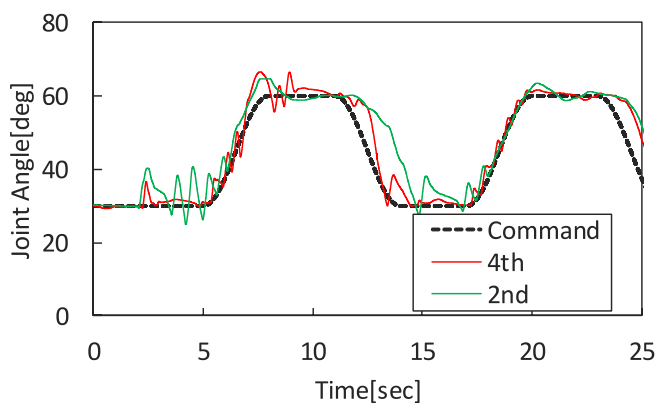

(c)

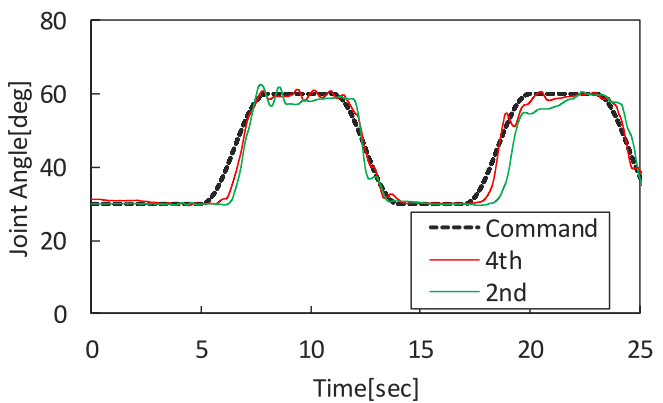

(d)

Fig. 15. Experimental results for the trapezoidal command for (a) Subject C, (b) Subject D, (c) Subject E, and (d) Subject F

results are in agreement with the simulation results. Because accurate modeling of $V^{\text {th }}$ was difficult, the resultant voltage $\hat{V}^{\text {th }}-V^{\text {th }}$ exhibited oscillation. However, the wide control bandwidth provided by the fourth-order full state feedback control drastically reduced this oscillation. Table 8 demonstrates drastic improvement of steady state RMSE (SSRMSE). Figure 15 also reveals faster responses and smaller oscillations for the proposed method. By examining the proposed method's response at approximately $13 \mathrm{~s}$ in Fig. 15(b), one can see that a rapid response occurs just after the delay for the command. This is attributed to a modeling error in $V^{t h}$, where $\hat{V}^{t h}$ was smaller than $V^{\text {th }}$. Figure 16 


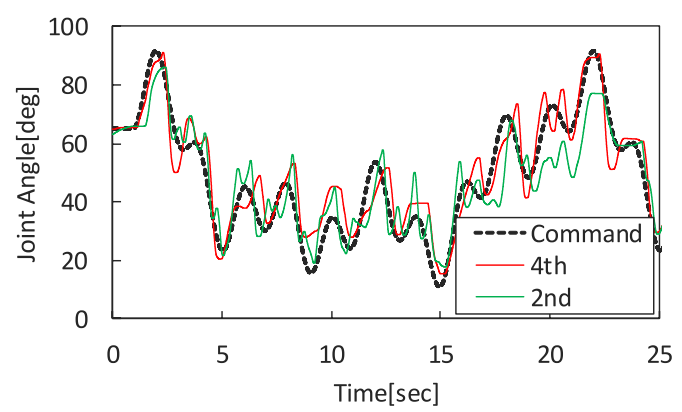

(a)

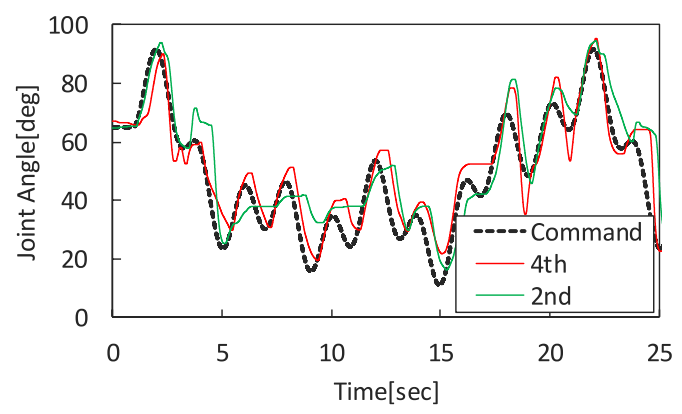

(b)

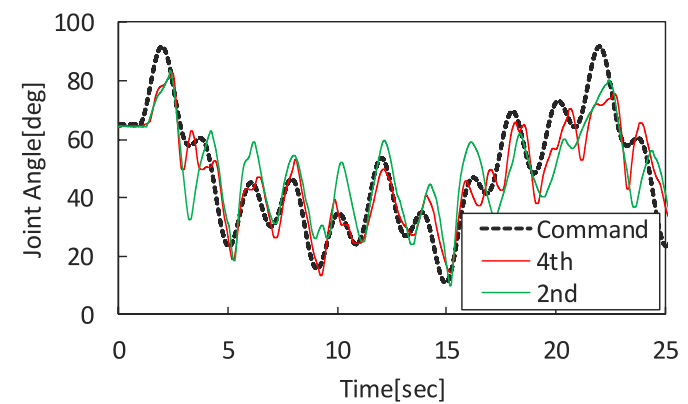

(c)

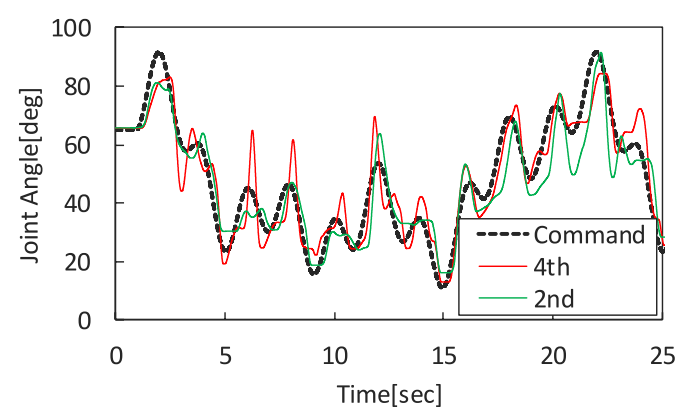

(d)

Fig. 16. Experimental results for the sine waves for (a) Subject C, (b) Subject D, (c) Subject E, and (d) Subject F

Table 8. SSRMSE [deg] values in step command. PD controller with 2 nd order model and proposed controller with 4 th order model

\begin{tabular}{|c|cc|}
\hline Subject & PD [deg] & Proposed [deg] \\
\hline C & 1.8 & 0.57 \\
D & 0.91 & 0.074 \\
E & 0.048 & 0.037 \\
F & 0.21 & 0.035 \\
\hline
\end{tabular}

reveals that the proposed method reduced tracking errors for the sine waves, although the errors for the sine waves were still larger than those for the step command. Because $V^{\text {th }}$ is dependent on joint angles, the time-varying command values require a precise angle-dependent model for $V^{\text {th }}$ for precise
Table 9. RMSE [deg] values for the trapezoidal command and sine waves. PD controller with 2 nd order model and proposed controller with 4th order model

\begin{tabular}{|c|c|c|c|c|}
\hline Subject & PD & Proposed & PD & Proposec \\
\hline & \multicolumn{2}{|c|}{ Trapezoid [deg] } & \multicolumn{2}{|c|}{ Sine wave [deg] } \\
\hline $\mathrm{C}$ & 4.21 & 2.68 & 8.74 & 7.40 \\
\hline D & 5.08 & 4.79 & 10.2 & 7.66 \\
\hline E & 6.27 & 4.09 & 11.4 & 7.96 \\
\hline $\mathrm{F}$ & 4.36 & 2.26 & 7.70 & 7.23 \\
\hline
\end{tabular}

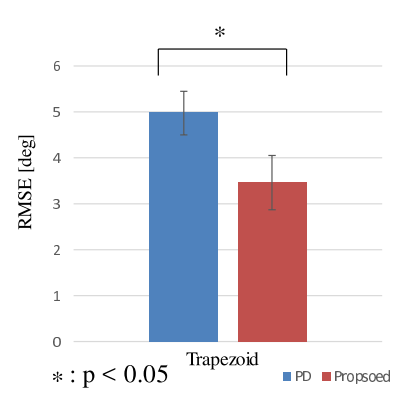

(a)

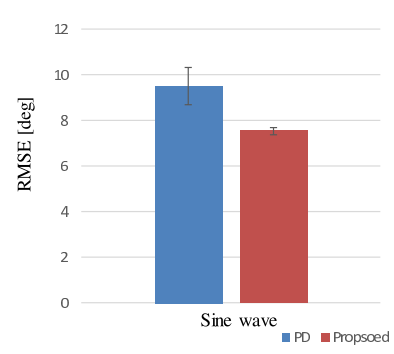

(b)
Fig. 17. Statistical comparison of average RMSE values: (a) trapezoidal command and (b) sine waves

control. However, such a model is difficult to implement, so control performance was degraded in this study. Therefore, the precise modeling and identification of $V^{\text {th }}$ is still an open problem. Based on RMSE, quantitative comparisons are presented in Table 9, demonstrating the superiority of the proposed method. A two sample paired t-test was performed to determine if the differences in test criteria were statistically significant at a $95 \%$ confidence level. Figure 17 reveals that the proposed method was statistically superior to the PD controller in terms of the trapezoidal command experiment (p-value $=0.0396)$. In contrast, we cannot confirm the statistical superiority of the proposed method for the sine wave experiment $(\mathrm{p}$-value $=0.0587)$. However, the $\mathrm{p}$-value is still relatively small.

Notably, frequency characteristics are typically examined to verify the bandwidth of a controller. However, frequency verification tests require long-duration experiments, resulting in muscle fatigue. Therefore, such experiments were not performed in this study. Phase recovery based on fourth-order feedback control was the primary objective of this study, rather than compensation for nonlinearities. Although FES nonlinearities were not explicitly addressed, the control performance of the proposed method was sufficient. This is because FES nonlinearities are related to slow dynamics, such as fatigue, which can be suppressed utilizing a simple feedback controller. Obviously, tracking performance can be further improved by adapting the jerk response feedback loop to more sophisticated controllers, such as the controller presented in ${ }^{(34)}$.

The method of reference ${ }^{(34)}$ improved the RMSE for sinusoidal commands by $19 \%$ on average compared to a linear PID controller. On the other hand, Table 9 shows that the proposed method improved the RMSE for the sinusoidal command by an average of $20 \%$ compared to the PD control. Note that the command value in this study was more difficult to follow than the command value in ${ }^{(34)}$ because the command value in this study includes higher frequency sinusoidal waves. Although a simple comparison is not possible 
due to the difference in the control system and the command value, the results suggest that the proposed method can be expected to provide the same level of control performance as the sophisticated controllers.

\section{Conclusion}

In this study, the time delay in FES was found to be negligible with respect to controller design because the phase delays caused by time delays are very small in frequency domains lower than natural angular frequencies. Furthermore, the transfer functions of musculoskeletal systems were found to be simple fourth-order linear systems, which simplifies controller design. Consequently, a full state feedback controller for FES was proposed to improve tracking performance. Jerk responses are required for the implementation of such a controller, so recently developed optical encoders that can effectively measure jerk responses were utilized in this study. The validity of the proposed model and controller was confirmed through simulations and experiments. A simple linear controller was implemented to improve tracking performance. The results of this study can easily be integrated with conventional controllers, such as SMC. Our next goal is to utilize the proposed model with high-resolution encoders to improve force and bilateral control.

\section{Acknowledgment}

This work was partly supported by JST, PRESTO Grant Number JPMJPR1755, and JSPS KAKENHI Grant Number $19 \mathrm{H} 04187$.

\section{References}

( 1 ) M. Ferrarin, F. Palazzo, R. Riener, and J. Quintern: "Model-based control of fes-induced single joint movements", IEEE Transactions on Neural Systems and Rehabilitation Engineering, Vol.9, No.3, pp.245-257 (2001)

( 2 ) C.L. Lynch and M.R. Popovic: "Functional electrical stimulation", IEEE Control Systems, Vol.28, No.2, pp.40-50 (2008)

( 3 ) M.M. Adamczyk and P.E. Crago: "Simulated feedforward neural network coordination of hand grasp and wrist angle in a neuroprosthesis", IEEE Transactions on Rehabilitation Engineering, Vol.8, No.3, pp.297-304 (2000)

( 4 ) M. Popovic, T. Thrasher, V. Zivanovic, J. Takaki, and V. Hajek: "Neuroprosthesis for retraining reaching and grasping functions in severe hemiplegic patients", Neuromodulation, Vol.8, No.1, pp.58-72 (2005)

( 5 ) Z. Matjacic and T. Bajd: "Arm-free paraplegic standing. part i: Control model synthesis and simulation”, IEEE Transactions on Rehabilitation Engineering, Vol.6, No.2, pp.125-138 (1998)

( 6 ) Z. Matjacic and T. Bajd: "Arm-free paraplegic standing. part ii: Experimental results", IEEE Transactions on Rehabilitation Engineering, Vol.6, No.2, pp.139-150 (1998)

( 7 ) W. Holderbaum, K. Hunt, and H. Gollee: " $H_{\infty}$ robust control design for unsupported paraplegic standing: experimental evaluation", Control Engineering Practice, Vol.10, No.11, pp.1211-1222 (2002)

( 8 ) N. Donaldson, T. Perkins, R. Fitzwater, D. Wood, and F. Middleton: "Fes cycling may promote recovery of leg function after incomplete spinal cord injury", Spinal Cord, Vol.38, No.11, pp.680-682 (2000)

( 9 ) T.J. Demchak, J.K. Linderman, W.J. Mysiw, R. Jackson, J. Suun, and S.T. Devor: "Effects of functional electric stimulation cycle ergometry training on lower limb musculature in acute sci individuals", Journal of Sports Science \& Medicine, Vol.4, No.3, pp.263-271 (2005)

(10) E. Ambrosini, S. Ferrante, G. Ferrigno, F. Molteni, and A. Pedrocchi: "Cycling induced by electrical stimulation improves muscle activation and symmetry during pedaling in hemiparetic patients", IEEE Transactions on Neural Systems and Rehabilitation Engineering, Vol.20, No.3, pp.320-330 (2012)

(11) T. Thrasher, H. Flett, and M. Popovic: "Gait training regimen for incomplete spinal cord injury using functional electrical stimulation", Spinal Cord, Vol.44, No.6, pp.357-361 (2006)

(12) S. Dosen, S. Member, and D.B. Popovic: "Moving-window dynamic optimization: Design of stimulation profiles for walking", IEEE Transactions on
Biomedical Engineering, Vol.56, No.5, pp.1298-1309 (2009)

(13) C.E. Bouton, A. Shaikhouni, N.V. Annetta, M.A. Bockbrader, D.A. Friedenberg, D.M. Nielson, G. Sharma, P.B. Sederberg, B.C. Glenn, W.J. Mysiw, A.G. Morgan, M. Deogaonkar, and A.R. Rezai: "Restoring cortical control of functional movement in a human with quadriplegia", Nature, Vol.533, pp.247-250 (2016)

(14) S. Qiu, W. Yi, J. Xu, H. Qi, J. Du, C. Wang, F. He, and D. Ming: "Eventrelated beta eeg changes during active, passive movement and functional electrical stimulation of the lower limb", IEEE Transactions on Neural Systems and Rehabilitation Engineering, Vol.24, No.2, pp.283-290 (2016)

(15) X. Zhao, Y. Chu, J. Han, and Z. Zhang: "SSVEP-based brain-computer interface controlled functional electrical stimulation system for upper extremity rehabilitation", IEEE Transactions on Systems, Man, and Cybernetics: System, Vol.46, No.7, pp.947-956 (2016)

(16) E. Tamaki, T. Miyaki, and J. Rekimoto: "Possessedhand: Techniques for controlling human hands using electrical muscles stimuli", in Proceedings of the SIGCHI Conference on Human Factors in Computing Systems, CHI'11, pp.543-552 (2011)

(17) P. Lopes, D. Yuksel, F. Guimbretiere, and P. Baudisch: "Muscle-plotter: an interactive system based on electrical muscle stimulation that produces spatial output", in Proceedings of the 29th Annual Symposium on User Interface Software and Technology, pp.207-217 (2016)

(18) P. Lopes, S. You, L.-P. Cheng, S. Marwecki, and P. Baudisch: "Providing haptics to walls \& heavy objects in virtual reality by means of electrical muscle stimulation", in Proceedings of the 2017 CHI Conference on Human Factors in Computing System, pp.1471-1482 (2017)

(19) T. Kitamura, S. Sakaino, and T. Tsuji: "Bilateral control using functional electrical stimulation", in Proceedings of the 41st Annual Conference of the IEEE Industrial Electronics, pp.2336-2341 (2015)

(20) T. Kitamura, N. Mizukami, H. Mizoguchi, S. Sakaino, and T. Tsuji: "Bilateral control in the vertical direction using functional electrical stimulation", IEEJ Journal of Industry Applications, Vol.5, No.5, pp.398-404 (2016)

(21) Y. Hasegawa, T. Kitamura, S. Sakaino, and T. Tsuji: "Bilateral control of elbow and shoulder joints using functional electrical stimulation between humans and robots", IEEE Access, Vol.8, pp.15792-15799 (2020)

(22) A.B. Hmed, T. Bakir, Y.M. Garnier, A. Sakly, R. Lepers, and S. Binczak: "An approach to a muscle force model with force-pulse amplitude relationship of human quadriceps muscles", Computers in Biology and Medicine, Vol.101, pp.218-228 (2018)

(23) N. Sharma, C.M. Gregory, and W.E. Dixon: "Predictor-based compensation for electromechanical delay during neuromuscular electrical stimulation", IEEE Transactions on Neural Systems and Rehabilitation Engineering, Vol.19, No.6, pp.601-611 (2011)

(24) S. Obuz, R.J. Downey, J.R. Klotz, and W.E. Dixon: "Unknown time-varying input delay compensation for neuromuscular electrical stimulation", in Proceedings of 2015 IEEE Conference on Control Applications, pp.365-370 (2015)

(25) K. Kurosawa, R. Futami, T. Watanabe, and N. Hoshimiya: "Joint angle control by fes using a feedback error learning controller", IEEE Transactions on Neural Systems and Rehabilitation Engineering, Vol.13, No.3, pp.359-371 (2005)

(26) G.-C. Chang, J.-J. Luh, G.-D. Liao, J.-S. Lai, C.-K. Cheng, B.-L. Kuo, and T.-S. Kuo: "A neuro-control system for the knee joint position control with quadriceps stimulation", IEEE Transactions on Rehabilitation Engineering, Vol.5, No.11, pp.2-11 (1997)

(27) P. Sampson, C. Freeman, S. Coote, and S. Demain: "Using functional electrical stimulation mediated by iterative learning control and robotics to improve arm movement for people with multiple sclerosis", IEEE Transactions on Neural Systems and Rehabilitation Engineering, Vol.24, No.2, pp.235-248 (2016)

(28) A. Ajoudani and A. Erfanian: "A neuro-sliding-mode control with adaptive modeling of uncertainty for control of movement in paralyzed limbs using functional electrical stimulation", IEEE Transactions on Biomedical Engineering, Vol.57, No.7, pp.1771-1780 (2009)

(29) A. Farhoud and A. Erfanian: "Fully automatic control of paraplegic fes pedaling using higher-order sliding mode and fuzzy logic control", IEEE Transactions on Neural Systems and Rehabilitation Engineering, Vol.22, No.3, pp.533-542 (2014)

(30) V. Nekoukar and A. Erfanian: "A decentralized modular control framework for robust control of fes-activated walker-assisted paraplegic walking using terminal sliding mode and fuzzy logic control", IEEE Transactions on Biomedical Engineering, Vol.59, No.10, pp.2818-2827 (2012)

(31) Q. Zhang, M. Hayashibe, P. Fraisse, and D. Guiraud: "Fes-induced torque prediction with evoked emg sensing for muscle fatigue tracking", IEEE/ASME Transactions on Mechatronics, Vol.16, No.5, pp.816-826 (2011)

(32) K. Matsui, Y. Hishii, K. Maegaki, Y. Yamashita, M. Uemura, H. Hirai, and 
F. Miyazaki: "Equilibrium-point control of human elbow-joint movement under isometric environment by using multichannel functional electrical stimulation", Frontiers in Neuroscience, Vol.8, No.164, pp.1-9 (2014)

(33) K.J. Hunt, M. Munih, N. de N. Donaldson, and F.M.D. Barr: "Investigation of the hammerstein hypothesis in the modeling of electrically stimulated muscle", IEEE Transactions on Biomedical Engineering, Vol.45, No.8, pp.9981009 (1998)

(34) N. Alibeji, N. Kirsch, B.E. Dicianno, and N. Sharma: "A modified dynamic surface controller for delayed neuromuscular electrical stimulation", IEEE/ASME Transactions on Mechatronics, Vol.22, No.4, pp.1755-1764 (2017)

(35) K. Yuki, T. Murakami, and K. Ohnishi: "Vibration control of a 2 mass resonant system by the resonance ratio control", IEEJ Transactions on Industry Applications, Vol.113, No.10, pp.1162-1169 (1993)

(36) Y. Hori, H. Sawada, and Y. Chun: "Slow resonance ratio control for vibration suppression and disturbance rejection in torsional system", IEEE Transactions on Industrial Electronics, Vol.46, No.1, pp.162-168 (1999)

(37) K. Tsuda, T. Sakuma, K. Umeda, S. Sakaino, and T. Tsuji: "Resonancesuppression control for electro-hydrostatic actuator as two-inertia system", IEEJ Journal of Industry Applications, Vol.6, No.5, pp.320-327 (2017)

(38) K. Araake, S. Sakaino, and T. Tsuji: "Design of resonance ratio control with relative position information for two-inertia system", in Proceedings of 2019 IEEE/ASME International Conference on Advanced Intelligent Mechatronics, pp.648-653 (2019)

(39) K. Szabat and T. Orlowska-Kowalska: "Vibration suppression in a two-mass drive system using pi speed controller and additional feedbacks-comparative study", IEEE Transactions on Industrial Electronics, Vol.54, No.2, pp.11931206 (2007)

(40) S.E. Saarakkala and M. Hinkkanen: "State-space speed control of two-mass mechanical systems: Analytical tuning and experimental evaluation", IEEE Transactions on Industry Applications, Vol.50, No.5, pp.3428-3437 (2014)

(41) R. Oboe and D. Pilastro: "Use of load-side mems accelerometers in servo positioning of two-mass-spring systems", in Proceedings of Proceedings of the 33rd Annual Conference of the IEEE Industrial Electronics, pp.4603-4608 (2015)

(42) K. Sakata, H. Asaumi, K. Hirachi, K. Saiki, and H. Fujimoto: "Self resonance cancellation techniques for a two-mass system and its application to a largescale stage", IEEJ Journal of Industry Applications, Vol.3, No.6, pp.455-462 (2014)

(43) M. Aoki, H. Fujimoto, Y. Hori, and T. Takahashi: "Robust resonance suppression control based on self resonance cancellation disturbance observer and application to humanoid robot", in Proceedings of 2013 IEEE International Conference on Mechatronics, pp.623-628 (2013)

(44) T. Sakuma, K. Tsuda, K. Umeda, S. Sakaino, and T. Tsuji: "Modeling and resonance suppression control for electro-hydrostatic actuator as a two-mass resonant system", Journal of Advanced Robotics, pp.1-11 (2017)

(45) S. Yamada, K. Inukai, H. Fujimoto, K. Omata, Y. Takeda, and S. Makinouchi: "Proposal of self resonance cancellation control without using drive-side information", in Proceedings of the 41st Annual Conference of the IEEE Industrial Electronics, pp.783-788 (2015)

(46) T. Yamazaki, S. Sakaino, and T. Tsuji: "Estimation and kinetic modeling of human arm using wearable robot arm", Electrical Enginnering in Japan, Vol.199, No.3, pp.57-67 (2017)

(47) T. Kitamura, S. Sakaino, and T. Tsuji: "Estimation of relationship between stimulating current and exerted force considering muscle length", in 5th IEEJ International Workshop on Sensing, Actuation, Motion Control, and Optimization (2019)
Sho Sakaino (Senior Member) received his B.E. degree in system

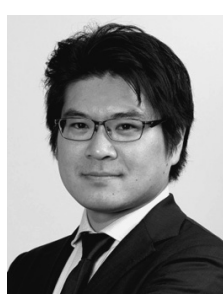
design engineering and M.E. and Ph.D. degrees in integrated design engineering from Keio University, Yokohama, Japan, in 2006, 2008, and 2011 respectively. He was an assistant professor at Saitama University from 2011, to 2019. Since 2019, he has been an associate professor at University of Tsukuba. His research interests include mechatronics, motion control, robotics, and haptics. He received the IEEJ Industry Application Society Distinguished Transaction Paper Award in 2011 and 2020. He also received the RSJ Advanced Robotics Excellent Paper Award in 2020.

Tomoya Kitamura (Student Member) received his B.E. and M.E. de-

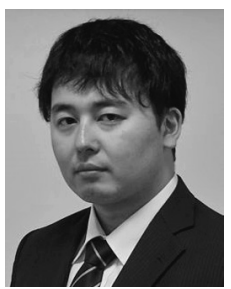
grees in electrical and electronic systems engineering from Saitama University, Saitama, Japan, in 2015 and 2017, respectively. He is currently a $\mathrm{Ph} . \mathrm{D}$. candidate. $\mathrm{He}$ is also a research fellow (DC2) of Japan Society for the Promotion of Science from 2018. His research interests include mechatronics and biomedical engineering.

Naoto Mizukami (Non-member) received his B.E. and M.E. degrees

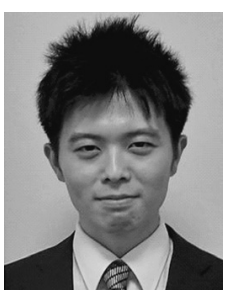
in electrical and electronic systems engineering from Saitama University, Saitama, Japan, in 2016 and 2018, respectively. He currently works at Central Japan Railway Company.

Toshiaki Tsuji (Senior Member) received his B.E. degree in system

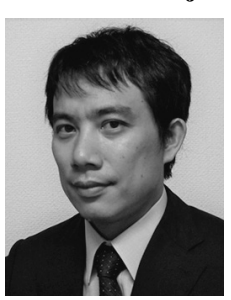
design engineering and M.E. and Ph.D. degrees in integrated design engineering from Keio University, Yokohama, Japan, in 2001, 2003, and 2006, respectively. He was a Research Associate in the Department of Mechanical Engineering, Tokyo University of Science, from 2006 to 2007. He is currently an Associate Professor in the Department of Electrical and Electronic Systems, Saitama University, Saitama, Japan. His research interests include motion control, haptics and rehabilitation robots. Dr. Tsuji received the FANUC FA and Robot Foundation Original Paper Award in 2007 and 2008, respectively. He also received the RSJ Advanced Robotics Excellent Paper Award and the IEEJ Industry Application Society Distinguished Transaction Paper Award in 2020 . 